\title{
CALCULATION OF PRICE EQUILIBRIA FOR UTILITY FUNCTIONS OF THE HARA CLASS
}

\author{
By MARKuS LIENHARD
}

Université de Lausanne

\begin{abstract}
We explicitly calculate price equilibria for power and logarithmic utility functions which-together with the exponential utility functions-form the so-called HARA (Hyperbolic Absolute Risk Aversion) class.

A price equilibrium is economically admissible in the market which is a closed system. Furthermore it is on the one side individually optimal for each participant of the market (in the sense of maximal expected utility), on the other side it is a Pareto optimum and thus collectively optimal for the market as a whole.
\end{abstract}

\section{KEY WORDS}

Risk exchange, Price equilibrium, Utility function, Pareto optimality, Risk aversion.

\section{INTRODUCTION}

This paper follows up BühLMANN (1980). First we repeat some of the notions and results of BÜHLMANN (1980).

The market consists of $n$ participants ("decision makers"), numbered from 1 to $n$, typically buyers of direct insurance, insurers, reinsurers.

Each participant $i(1 \leqslant i \leqslant n)$ is characterized by

$w_{i}: \quad$ Initial wealth

$u_{i}(x)$ : Utility function (as usual $u_{i}^{\prime}>0, u_{i}^{\prime \prime} \leqslant 0$ )

$X_{i}(\omega)$ : Initial portfolio (random variable)

$Y_{i}(\omega)$ : Payment (reimbursement) (random variable) bought for the premium $E\left[\phi Y_{i}\right]$.

$\phi(\omega)$ is a price function.

Remark. All random variables are given on a probability space $(\Omega, \mathfrak{A}, P)$ and are expected to be sufficiently regular in all cases. Particularly the appearing expected values should always exist.

The "exchange variable" $Y_{i}$, bought by $i$, represents the payment which $i$ obtains from the other participants. So the $Y_{1}, \ldots, Y_{n}$ must satisfy the economic admissibility condition of the market

$$
\sum_{i=1}^{n} Y_{i}(\omega)=0 \quad \forall \omega \in \Omega
$$

ASTIN BULLETIN Vol. 16, $\mathrm{S}$ 
After having bought $Y_{i}$ and paid for that the amount $E\left[\phi Y_{i}\right], i$ has a modified portfolio

$$
X_{i}-Y_{i}+E\left[\phi Y_{i}\right]
$$

We call each such vector of random variables

$$
Y=\left(Y_{1}, \ldots, Y_{n}\right),
$$

satisfying (1), a risk exchange in the market. Furthermore a price equilibrium of the market is a pair

$$
\left(\tilde{\phi}, \tilde{\boldsymbol{Y}}=\left(\tilde{Y}_{1}, \ldots, \tilde{Y}_{n}\right)\right)
$$

satisfying the two conditions

(I) $\sum_{i=1}^{n} \tilde{Y}_{i}=0$, i.e., $\tilde{\boldsymbol{Y}}$ is a risk exchange

(II) $\forall i: \tilde{Y}_{i}$ maximizes the function

$$
Y_{i} \mapsto E\left[u_{i}\left(w_{i}-X_{i}+Y_{i}-E\left[\tilde{\phi} Y_{i}\right]\right)\right] .
$$

$\tilde{\boldsymbol{Y}}$ is called an equilibrium risk exchange whereas $\tilde{\phi}$ is an equilibrium price.

Condition (II) implies that each participant evaluates his "situation" according to the principle of expected utility. In BüHLMANN (1980) is shown that (II) is equivalent to the condition (III)

$$
\text { (III) } \forall i: \quad \begin{gathered}
u_{i}^{\prime}\left(w_{i}-X_{i}(\omega)+\tilde{Y}_{i}(\omega)-E\left[\tilde{\phi} \tilde{Y}_{i}\right]\right)=\tilde{\phi}(\omega) \underbrace{E\left[u_{i}^{\prime}\left(w_{i}-X_{i}+\tilde{Y}_{i}-E\left[\tilde{\phi} \tilde{Y}_{i}\right]\right)\right]}_{=: C_{i}} \text { for almost all } \omega \in \Omega .
\end{gathered}
$$

Hence an equilibrium $(\tilde{\phi}, \tilde{\boldsymbol{Y}})$ is also characterized by (I) and (III).

Each vector of random variables

$$
\boldsymbol{Z}=\left(Z_{1}, \ldots, Z_{n}\right),
$$

satisfying

$$
\sum_{i=1}^{n} Z_{i}(\omega)=\sum_{i=1}^{n} X_{i}(\omega) \quad \forall \omega \in \Omega,
$$

is an admissible modification of the initial portfolios $\left(X_{1}, \ldots, X_{n}\right)$. An admissible modification $Z$ is Pareto optimal if there exists no admissible modification $Z^{*}$ with

$$
\forall i: \quad E\left[u_{i}\left(w_{i}-Z_{i}^{*}\right)\right]>E\left[u_{i}\left(w_{i}-Z_{i}\right)\right] .
$$

The admissible modification $\tilde{\boldsymbol{Z}}$,

$$
\tilde{Z}_{i}:=X_{i}-\tilde{Y}_{i}+E\left[\tilde{\phi} \tilde{Y}_{i}\right] \quad(1 \leqslant i \leqslant n),
$$

induced by the equilibrium $(\tilde{\phi}, \tilde{\boldsymbol{Y}})$, is Pareto optimal. For this see BüHLMaNN (1980) ((III) is just Borch's condition, necessary and sufficient for Pareto optimality).

A further and immediate consequence of the equivalence (II) $\Leftrightarrow$ (III) is that the equilibrium price is automatically "normalized":

$$
E[\tilde{\phi}]=1 \text {. }
$$


It follows from (4) that $\tilde{\boldsymbol{Y}}$ is determined only up to an additive constant: $\left(\tilde{Y}_{1}+c_{1}, \ldots, \tilde{Y}_{n}+c_{n}\right)$ is an equilibrium exchange if $\left(\tilde{Y}_{1}, \ldots, \tilde{Y}_{n}\right)$ is one and if $\sum_{i=1}^{n} c_{i}=0$.

Hence we can put without loss of generality

$$
E\left[\tilde{\phi} \tilde{Y}_{i}\right]=0 \quad(1 \leqslant i \leqslant n) \quad\left(c_{i}:=-E\left[\tilde{\phi} \tilde{Y}_{i}\right]\right) .
$$

Then (3) becomes

$$
\tilde{Z}_{i}=X_{i}-\tilde{Y}_{i}
$$

Remark. Observe that (2) (as well as (1)) is a "clearing condition" in the market which is a closed system.

REMARK. The existence of an equilibrium is dealt with in BüHLMANN (1984) (which follows up BüHLMANN (1980)).

\section{EXPLICIT CALCULATIONS}

We explicitly calculate equilibria where the utility functions $u_{i}$ are

(a) power functions

(b) logarithmic functions

(c) exponential functions.

Observe in the following that our considered utility functions $u_{i}$ are indeed increasing $\left(u_{i}^{\prime}>0\right)$ and concave $\left(u_{i}^{\prime \prime} \leqslant 0\right)$.

Hence: Given: $w_{i}, X_{i}, u_{i}(1 \leqslant i \leqslant n)$

Wanted: Pair $(\tilde{\phi}, \tilde{\boldsymbol{Y}})\left(\tilde{\boldsymbol{Y}}=\left(\tilde{Y}_{1}, \ldots, \tilde{Y}_{n}\right)\right)$ satisfying (I), (III) and (5). We will see that the $\tilde{Y}_{i}$ have the form

$$
\tilde{Y}_{i}=X_{i}-\gamma_{i} \sum_{i=1}^{n} X_{i}-\delta_{i}
$$

where the $\gamma_{i}$ 's and $\delta_{i}$ 's are constants with

$$
\gamma_{i} \geqslant 0, \quad \sum_{i=1}^{n} \gamma_{i}=1 ; \quad \sum_{i=1}^{n} \delta_{i}=0 .
$$

We can also write in the " $Z$-language" (as in (6))

$$
\tilde{Z}_{i}=X_{i}-\tilde{Y}_{i}=\gamma_{i} \sum X_{i}+\delta_{i} \text {. }
$$

Hence according to the modified portfolio $\tilde{Z}_{i}$ participant $i$ pays the quota $\gamma_{i}$ of the total claims $\sum X_{i}$ as well as a deterministic side payment $\delta_{i}$. This result does not surprise, see e.g., BüHLMANN and Jewell (1979).

(a) Power Case

We consider

$$
u_{i}(x):=\mp\left(a_{i+}{ }^{\mp} x\right)^{c} \quad \text { for }\left\{\begin{array}{r}
c>1 \\
0<c<1 \\
c<0
\end{array} \quad(1 \leqslant i \leqslant n) .\right.
$$


Observe that the $u_{i}$ 's are concave indeed $\left(u_{i}^{\prime \prime} \leqslant 0\right)$. The domain of $u_{i}$ is given by the requirement that $u_{i}^{\prime}>0$. The $a_{i}$ 's are constants.

TheOREM. The equilibrium $\left(\tilde{\phi}, \tilde{Y}=\left(\tilde{Y}_{1}, \ldots, \tilde{Y}_{n}\right)\right)$ can be explicitly calculated and has the form

$$
\begin{gathered}
\tilde{\phi}(\omega)=\frac{\left(\sum a_{i} \mp \sum w_{i} \pm \sum X_{i}(\omega)\right)^{c-1}}{E\left[\left(\sum a_{i} \mp \sum w_{i} \pm \sum X_{i}\right)^{c-1}\right]}\left\{\begin{array}{c}
c>1 \\
0 \neq c<1
\end{array}\right. \\
X_{i}(\omega)-\tilde{Y}_{i}(\omega)=\gamma_{i} \cdot \sum X_{i}(\omega)+\delta_{i} \quad(1 \leqslant i \leqslant n)
\end{gathered}
$$

where

$$
\left\{\begin{array}{l}
\gamma_{i}=\frac{E\left[\left(\sum a_{i} \mp \sum w_{i} \pm \sum X_{i}\right)^{c-1}\left(a_{i} \mp w_{i} \pm X_{i}\right)\right]}{E\left[\left(\sum a_{i} \mp \sum w_{i} \pm \sum X_{i}\right)^{c}\right]} \\
\delta_{i}= \pm\left(\sum a_{i} \mp \sum w_{i}\right) \cdot \gamma_{i} \mp\left(a_{i} \mp w_{i}\right) .
\end{array}\right.
$$

Observe that it is indeed

$$
\sum \gamma_{i}=1, \quad \sum \delta_{i}=0
$$

REMARK. The case of three signs is for $\left\{\begin{array}{c}c>1 \\ 0<c<1 \text {, whereas we put two signs } \\ c<0\end{array}\right.$ for $\left\{\begin{array}{r}c>1 \\ 0 \neq c<1\end{array}\right.$.

Proof. With (7) we have

$$
u_{i}^{\prime}(x)=(\stackrel{+}{ \pm} c)\left(a_{i}^{\mp} x\right)^{c-1}
$$

and it follows from (III) and (5)

$$
(\underset{ \pm}{\ddagger} c)\left(a_{i} \mp\left[w_{i}-X_{i}+\tilde{Y}_{i}\right]\right)^{c-1}=\tilde{\phi} C_{i}
$$

or

$$
a_{i+} \mp w_{i} \pm X_{i+}^{\mp} \tilde{Y}_{i}=\tilde{\phi}^{1 /(c-1)}\left(\frac{C_{i}}{ \pm \underline{ \pm}}\right)^{1 /(c-1)},
$$

and after summation over all $i$ it follows with (I)

$$
\sum a_{i+}^{\mp} \sum w_{i} \pm \sum X_{i}=\tilde{\phi}^{1 /(c-1)} \sum\left(\frac{C_{i}}{ \pm c}\right)^{1 /(c-1)} .
$$

When we solve the last equation for $\tilde{\phi}$, we obtain with the "norming condition" (4) the formula (8).

We see that in the sequel it is enough to distinguish only two cases for the signs, concerning $\tilde{\phi}$. 
We determine now $\tilde{Y}_{i}$. For this we multiply both sides of (11) with $\tilde{\phi}$ and then we take the expected value (so we take on both sides $E[\tilde{\phi} \cdot]$ ). We obtain with (5)

$$
E\left[\tilde{\phi}\left(a_{i} \mp w_{i} \pm X_{i}\right)\right]=E\left[\tilde{\phi} \tilde{\phi}^{1 /(c-1)}\right]\left(\frac{C_{i}}{ \pm c}\right)^{1 /(c-1)} .
$$

(11) divided by (12) gives

$$
\frac{a_{i} \mp w_{i} \pm X_{i} \mp \tilde{Y}_{i}}{E\left[\tilde{\phi}\left(a_{i} \mp w_{i} \pm X_{i}\right)\right]}=\frac{\tilde{\phi}^{1 /(c-1)}}{E\left[\tilde{\phi} \tilde{\phi}^{1 /(c-1)}\right]}
$$

or

$$
\frac{a_{i} \mp w_{i} \pm X_{i} \mp \tilde{Y}_{i}}{\tilde{\phi}^{1 /(c-1)}}=\frac{E\left[\tilde{\phi}\left(a_{i} \mp w_{i} \pm X_{i}\right)\right]}{E\left[\tilde{\phi} \tilde{\phi}^{1 /(c-1)}\right]} .
$$

We insert $\tilde{\phi}$ of (8) in (13) and obtain after simplication (the denominator of (8) disappears)

$$
\frac{a_{i} \mp w_{i} \pm X_{i} \mp \tilde{Y}_{i}}{\sum a_{i} \mp \sum w_{i} \pm \sum X_{i}}=\frac{E\left[\left(\sum a_{i} \mp \sum w_{i} \pm \sum X_{i}\right)^{c-1}\left(a_{i} \mp w_{i} \pm X_{i}\right)\right]}{E\left[\left(\sum a_{i} \mp \sum w_{i} \pm \sum X_{i}\right)^{c}\right]}
$$

We see from this that

$$
\tilde{Z}_{i}=X_{i}-\tilde{Y}_{i}
$$

is of the type

$$
\gamma_{i} \sum X_{i}+\delta_{i}
$$

where $\gamma_{i}$ and $\delta_{i}$ have indeed the form (9).

Some special cases of formula (8)

$c:=2$ gives the quadratic utility functions

$$
u_{i}(x)=-\left(a_{i}-x\right)^{2} \quad\left(x \leqslant a_{i}\right) \quad(1 \leqslant i \leqslant n) .
$$

We then have for the "quadratic price"

$$
\begin{gathered}
\tilde{\phi}(\omega)=\left(\sum a_{i}-\sum w_{i}+\sum X_{i}(\omega)\right) / E\left[\sum a_{i}-\sum w_{i}+\sum X_{i}\right] . \\
c:=\frac{1}{2} \text { fournishes utility functions of square root type: } \\
u_{i}(x)=\sqrt{a_{i}+x} \quad\left(x \geqslant-a_{i}\right) \quad(1 \leqslant i \leqslant n) .
\end{gathered}
$$

In this case we have

$$
\begin{gathered}
\tilde{\phi}(\omega)=\frac{1}{\sqrt{\sum a_{i}+\sum w_{i}-\sum X_{i}(\omega)}} / E\left[\frac{1}{\sqrt{\sum a_{i}+\sum w_{i}-\sum X_{i}}}\right] . \\
c:=-1 \text { finally leads to hyperbolic utility functions } \\
u_{i}(x)=-\frac{1}{a_{i}+x} \quad\left(x>-a_{i}\right) \quad(1 \leqslant i \leqslant n) .
\end{gathered}
$$


Then the equilibrium price becomes

$$
\tilde{\phi}(\omega)=\frac{1}{\left(\sum a_{i}+\sum w_{i}-\sum X_{i}(\omega)\right)^{2}} / E\left[\frac{1}{\left(\sum a_{i}+\sum w_{i}-\sum X_{i}\right)^{2}}\right] .
$$

(b) Logarithmic Case

$$
u_{i}(x):=\log \left(a_{i}+x\right) \quad\left(x>-a_{i}\right) \quad(1 \leqslant i \leqslant n) .
$$

The derivatives $u_{i}^{\prime}$-because of the property (III) essential for the calculation of the equilibrium - which appear here,

$$
u_{i}^{\prime}(x)=\left(a_{i}+x\right)^{-1},
$$

belong to the same type of functions like those in the power case. If we compare (14) with (10) we obtain from (8) and (9) directly the formulas

$$
\begin{gathered}
\tilde{\phi}(\omega)=\frac{\left(\sum a_{i}+\sum w_{i}-\sum X_{i}(\omega)\right)^{-1}}{E\left[\left(\sum a_{i}+\sum w_{i}-\sum X_{i}\right)^{-1}\right]} \\
X_{i}(\omega)-\tilde{Y}_{i}(\omega)=\gamma_{i} \sum X_{i}(\omega)+\delta_{i} \quad(1 \leqslant i \leqslant n)
\end{gathered}
$$

where

$$
\begin{aligned}
\gamma_{i} & =E\left[\frac{a_{i}+w_{i}-X_{i}}{\sum a_{i}+\sum w_{i}-\sum X_{i}}\right] \\
\delta_{i} & =-\left(\sum a_{i}+\sum w_{i}\right) \gamma_{i}+\left(a_{i}+w_{i}\right) .
\end{aligned}
$$

Hence the logarithmic case is a special case of the power case.

Observe again

$$
\sum \gamma_{i}=1, \quad \sum \delta_{i}=0
$$

(c) Exponential Case

Let's finally consider the well known exponential utility functions

$$
u_{i}(x)=\frac{1}{a_{i}}\left(1-e^{-a_{i} x}\right) \quad(x \in \mathbb{R}) \quad(1 \leqslant i \leqslant n)
$$

and for completeness cite BüHLMANN's (1980) formulas. The parameter $a_{i}>0$ stands here for the risk aversion of $i$. With the abbreviation

$$
\frac{1}{a}:=\sum \frac{1}{a_{i}}
$$

it is

$$
\tilde{\phi}(\omega)=\exp \left(a \sum X_{i}(\omega)\right) / E\left[\exp \left(a \sum X_{i}\right)\right]
$$




$$
\begin{gathered}
\gamma_{i}=\frac{1 / a_{i}}{1 / a} \\
\delta_{i}=E\left[\exp \left(a \sum X_{i}\right)\left(X_{i}-\gamma_{i} \sum X_{i}\right)\right] / E\left[\exp \left(a \sum X_{i}\right)\right]
\end{gathered}
$$

(and $\sum \gamma_{i}=1, \sum \delta_{i}=0$ ).

\section{REFERENCES}

Bühlmann, H. (1980) An Economic Premium Principle. ASTIN Bulletin 11 (1), 52-60.

BühlmanN, H. (1984) The General Economic Premium Principle. ASTIN Bulletin 14 (1).

BühlmanN, H. and Jewell, W. S. (1979) Optimal Risk Exchanges. ASTIN Bulletin 10 (3), 249.

\section{MARCus Lienhard}

Université de Lausanne, HEC, CH-1015 Lausanne, Switzerland 